\title{
Systemic overexpression of the 11 $\beta$-HSD1 promotes endoplasmic reticulum stress in multiple tissues and the development of metabolic syndrome in mice
}

\author{
CHENXIAO LI ${ }^{1}$, JIHAN XIA ${ }^{1}$, WENJUAN ZHU ${ }^{1}$, LEILEI XIN ${ }^{1}$, CUIPING AN ${ }^{1}$, SHULIN YANG ${ }^{1}$ and KUI LI ${ }^{1,2}$ \\ ${ }^{1}$ State Key Laboratory of Animal Nutrition, Institute of Animal Sciences, Chinese Academy of \\ Agricultural Sciences, Beijing 100193; ${ }^{2}$ Agricutural Genomes Institute at Shenzhen, Chinese \\ Academy of Agricultural Sciences, Shenzhen, Guangdong 518120, P.R. China
}

Received November 11, 2016; Accepted July 11, 2017

DOI: $10.3892 / \mathrm{mmr} .2017 .7530$

\begin{abstract}
Glucocorticoids are associated with lipid metabolism and their abnormal expression has an important function in the development of metabolic syndrome. The $11 \beta$-hydroxysteroid dehydrogenase type 1 (11 $\beta$-HSD1) is a metabolic enzyme of glucocorticoids and may be a potential drug target for the treatment of metabolic syndrome. However, the association between the systemic expression of $11 \beta$-HSD1 and metabolic syndrome remains to be elucidated. The present study used a cytomegalovirus promoter to obtain mice that systemically overexpressed the $11 \beta$-HSD1 gene. The transgenic mice and negative control groups received a high-fat diet at the age of 10 weeks in order to induce metabolic syndrome and this diet was continued for 12 weeks. Several indicators, including body weight, blood glucose, glucose tolerance and insulin resistance, were monitored in vivo. In addition, the protein expression levels of 11 $\beta$-HSD1 and DNA damage inducible transcript 3 were detected and the histopathology of important tissues for metabolic syndrome were analyzed. The current findings revealed that the body weights of transgenic mice were significantly higher compared with the control group before and during the periods of high fat diet induction. Transgenic mice also exhibited significantly impaired glucose tolerance, insulin resistance, endoplasmic reticulum stress and increased metabolic syndrome-associated biochemical indicators in the blood and severely impaired liver and kidney functions. The present study successfully established a $11 \beta-H S D 1$ systemic overexpression mouse model that exhibited typical characteristics of
\end{abstract}

Correspondence to: Dr Shulin Yang, State Key Laboratory of Animal Nutrition, Institute of Animal Sciences, Chinese Academy of Agricultural Sciences, 2 Yuanmingyuan West Road, Beijing 100193, P.R. China

E-mail: yangshulin@caas.cn

Key words: 11 $\beta$-HSD1, systemic overexpression, endoplasmic reticulum stress, hepatic lipidosis, metabolic syndrome metabolic syndrome and may be useful for future studies of metabolic syndrome.

\section{Introduction}

Metabolic syndrome consists of a group of complex metabolic disorders associated with the metabolism of carbohydrates, lipids and proteins. According to the definition of the International Diabetes Federation, patients who meet at least three of the following criteria are diagnosed with metabolic syndrome: i) Abdominal obesity; ii) hypertension, hyperlipemia, hypercholesterolemia; and iii) reduced high-density lipoprotein cholesterol levels (1). In 2005, >25\% the total world population was diagnosed with metabolic syndrome (2). In $2012,>1 / 3$ of the adult population and half of the population above 60 years of age have been diagnosed with metabolic syndrome in the United States (3). The negative impact of metabolic syndrome is primarily due to its influence on other diseases. In patients with metabolic syndrome, the risk of diabetes mellitus is increased 5-fold, the risk of cardiovascular diseases 3-fold, the risk of cardiovascular death 2-fold and the overall risk of death is increased 1.5-fold (4,5).

Glucocorticoids are involved in the regulation of carbohydrate, lipid, protein, water and salt metabolism and have important physiological functions (6-8). Previous studies have confirmed that excessive glucocorticoids may induce insulin resistance, inhibit glucose absorption and reduce insulin release, thus increasing the possibility developing metabolic syndrome (6). In patients with metabolic syndrome, the glucocorticoid levels in blood circulation are normal; however, the glucocorticoid levels in local tissues are increased. 11 $\beta$-hydroxysteroid dehydrogenase type 1 $(11 \beta-H S D 1)$ is a metabolic reductase and dehydrogenase that converts glucocorticoids between active cortisol and inactive cortisone (7). Therefore, $11 \beta$-HSD1 is a local amplifier of glucocorticoid functions (9). Alberts et al (8) confirmed that the $11 \beta$-HSD1-specific inhibitor BTV2733 reduced the levels of fasting blood glucose, insulin, the concentrations of blood cholesterol, free fatty acids and triglycerides in mice. Additionally, 11 $\beta$-HSD1 knockout mice exhibited weakened insulin resistance and gluconeogenesis reaction, enhanced 
glucose tolerance and improved lipid distribution (10). Masuzaki et al (11) and Paterson et al (12) already confirmed that the tissue-specific overexpression of 11 $\beta$-HSD1 in the liver and fat induced metabolic syndrome characteristics in mice. However, these two transgenic models could not reflect the overall effect of the systemic expression of the 11 $\beta$-HSD1 gene in patients with metabolic syndrome. Therefore, the present study established transgenic mice that systemically expressed the $11 \beta$-HSD1 gene and fed these mice with a high-fat diet to induce metabolic syndrome. The current findings revealed that transgenic mice exhibited metabolic syndrome characteristics and pathological features in key tissues.

\section{Materials and methods}

Experimental animals. The porcine $11 \beta$-HSD1 gene was ligated into the $\mathrm{T}$ vector and then ligated into the pcDNA3.1 plasmid (donated by Dr Li Li, Institute of Zoology, Chinese Academy of Sciences) using double-enzyme digestion. The digestion sites were NheI and PmeI. The constructed vector plasmid was injected into $10 \mathrm{C} 57 \mathrm{BL} / 6 \mathrm{~L}$ mice (Institute of Laboratory Animal Science, Chinese Academy of Medical Sciences; Beijing, China) via pronuclear microinjection to obtain transgenic mice. This was conducted at the Institute of Laboratory Animal Science, Chinese Academy of Medical Sciences. Matched C57 mice were purchased from Beijing Vital River Laboratory Animal Technology Co., Ltd. (Beijing, China). The offspring of transgenic founders were divided into the transgenic group and the negative control group and each group consisted of 5 mice. High-fat diets were purchased from Beijing Biopike Biotechnology Co., Ltd. (Beijing, China) with fat content of 58\%. All mice received humane care according to the criteria outlined in the 'Guide for the Care and Use of Laboratory Animals, Institute of Animal Sciences, Chinese Academy of Agricultural Sciences, Beijing, China.' The procedures were approved by the Institutional Animal Care and Use Committee of Chinese Academy of Agricultural Sciences. The mice were housed under a 12-h light/dark cycle with access to food and water ad libitum.

Following the termination of high-fat diet feeding, the mice in the two groups were anaesthetized by intraperitoneal injection of sodium pentobarbital (Sigma-Aldrich, Shanghai, China) at a dose of $50 \mathrm{mg} / \mathrm{kg}$ body weight and subsequently decapitated. Blood samples were collected and pancreatic, liver, lipid, kidney and muscle tissues were immediately removed, rinsed with cold physiological saline, frozen in liquid nitrogen and stored at $-80^{\circ} \mathrm{C}$. Pieces of liver about $\sim 10 \mathrm{~mm}$ diameter were fixed in $4 \%$ paraformaldehyde at $4^{\circ} \mathrm{C}$ for histopathological studies.

Polymerase chain reaction (PCR) detection. At 2 weeks of age, the tail tips of the mice were cut to extract genomic DNA using the phenol-chloroform method (13). The PCR product was 598 bp long for the following primers: F 5'-CCCATA GTAACGCCAATA-3' and R, 5'-CTACTGCTATTCCGC AAA-3'. The reaction was conducted in a total volume of $20 \mu \mathrm{l}$ and performed for 35 cycles under the following conditions: Pre-denaturation at $95^{\circ} \mathrm{C}$ for $5 \mathrm{~min}$, denaturation at $95^{\circ} \mathrm{C}$ for $30 \mathrm{sec}$, annealing at $58^{\circ} \mathrm{C}$ for $30 \mathrm{sec}$ and extension at $72^{\circ} \mathrm{C}$ for $30 \mathrm{sec}$ and a final extension at $72^{\circ} \mathrm{C}$ for $5 \mathrm{~min}$. All reagents were obtained from Takara Biotechnology Co., Ltd. (Dalian, China). Following PCR, 1\% agarose gel electrophoresis (Biowest Regular Agarose G-10; Biowest, Hong Kong, China). was performed.

Body weight, glucose and insulin tolerance test. During the high-fat diet feeding period, the body weight of the mice was measured once every other week. At the end of the experiment, a glucose tolerance test was performed. Before the test, the mice were fasted for $12 \mathrm{~h}$ and an intraperitoneal injection of $20 \%$ glucose at a dose of $2 \mathrm{~g} / \mathrm{kg}$ body weight was then administered. Blood samples $(2 \mu \mathrm{l})$ were collected from the tail tips prior to injection and at 15, 30, 60, 90 and $120 \mathrm{~min}$ after injection to quantify the blood glucose levels. For the insulin tolerance test, the mice were fasted for $6 \mathrm{~h}$ prior to an intraperitoneal injection of insulin at a dose of $1 \mathrm{IU} / \mathrm{kg}$ body weight. Blood samples $(2 \mu \mathrm{l})$ were collected from tail tips prior to the injection and 15,30,60 and $90 \mathrm{~min}$ after the injection to measure the blood glucose levels. Glucose was purchased from Mallinckrodt Pharmaceuticals Ltd. (Staines-upon-Thames, UK) and insulin (Novolin) was purchased from Novo Nordisk (Bagsværd, Denmark). A Onetouch Ultra blood glucose meter and blood glucose test strips were obtained from LifeScan, Inc. (Milpitas, CA, USA).

Serological quantification. Biochemical parameters were analyzed using a AU480 automatic biochemistry analyzer (Olympus Corporation, Tokyo, Japan). Assay kits were purchased from InTec Products, Inc. (Xiamen, China). Alanine aminotransferase (ALT), aspartate aminotransferase (AST) and uric acid were analyzed using the dehydrogenase enzyme method, triglyceride and creatinine were analyzed using the oxidase enzyme method, high-density lipoprotein-cholesterol and low-density lipoprotein-cholesterol were analyzed using direct method and total cholesterol was analyzed using the enzyme method as previously described (14).

Western blotting. Total protein was extracted from tissues using a tissue total protein extraction reagent kit (Thermo Fisher Scientific, Inc., Waltham, MA, USA) and the total protein concentration was quantified using an enzyme-linked immunosorbent assay (ELISA) using a microplate reader (Spectra Max M5, Molecular Devices, LLC, Sunnyvale, CA, USA). Subsequently, the proteins were denatured and stored at $-20^{\circ} \mathrm{C}$. For each sample, $30 \mu \mathrm{g}$ of protein was separated using SDS-PAGE and the proteins were then transferred onto a nitrocellulose (NC) membrane. The NC membrane was blocked in 5\% non-fat milk. To quantitatively detect the $11 \beta$-HSD1 protein, a primary $11 \beta-H S D 1$ antibody (cat. no. ab83522; 1:1,000; Abcam, Cambridge, UK) was used at room temperature for $2 \mathrm{~h}$ and a horseradish peroxidase-labeled secondary goat anti-rabbit antibody (cat. no. ab6721; 1:5,000; Abcam) was added at room temperature for $40 \mathrm{~min}$. To assess endoplasmic reticulum stress, a DNA damage inducible transcript 3 primary antibody (DDIT3; cat. no. ab11419; 1:1,000; Abcam) was used for incubation at room temperature for $2 \mathrm{~h}$ and a secondary goat anti-mouse antibody (cat. no. ab6789; 1:5,000; Abcam) were added for incubation at room temperature for $40 \mathrm{~min}$. Following washing, Super Signal West Pico 
Chemiluminescent substrate (Thermo Fisher Scientific, Inc.) was used to detect the immunoblots.

Hematoxylin and eosin (HE) staining. Tissues were sectioned at a thickness of $4 \mu \mathrm{m}$, and the sectioned slides were warmed at $60^{\circ} \mathrm{C}$ for $1 \mathrm{~h}$, deparaffinized using xylene, rehydrated, stained with hematoxylin at room temperature for $2 \mathrm{~min}$, washed, differentiated, blued, stained with eosin at room temperature for $1 \mathrm{~min}$, and washed with water. The stained sections were dehydrated in 70, 80, 90, 95 and 100\% ethanol ( 2 min for each concentration), followed by clearing in xylene 1 and xylene 2 ( $5 \mathrm{~min}$ each), the sections were subsequently mounted in resin.

Statistical analysis. The differences of data between the two study groups were statistically analyzed using SPSS version 18.0 (SPSS, Inc., Chicago, IL, USA). One-way analysis of variance and the least significant difference post-hoc test were used for multiple comparisons. $\mathrm{P}<0.05$ was considered to indicate a statistically significant difference. Data are presented as the mean \pm standard deviation.

\section{Results}

Detection of positive transgenic mice. The founders of $11 \beta$-HSD1 transgenic mice and their offspring were detected using PCR. The positive F0 individuals had the band of $598 \mathrm{bp}$ (Fig. 1).

Significant increase in obesity of transgenic mice. The average body weight of transgenic mice was $32.07 \pm 2.43 \mathrm{~g}$ prior to feeding with high-fat diet and the negative control group weight was $26.29 \pm 1.09 \mathrm{~g}(\mathrm{P}<0.05)$. From week 2 of high-fat diet induction until the end of the induction experiment, transgenic mice were significantly more obese compared with control mice. In addition, the body weights of transgenic mice consistently increased, whereas the body weights of control mice plateaued at week 20 and subsequently decelerated (Fig. 2). Following induction, the average body weight of the transgenic group was 1.56 -fold higher compared with the control group, $54.22 \pm 5.90 \mathrm{~g}$ and $40.46 \pm 3.23 \mathrm{~g}(\mathrm{P}<0.05)$.

Biochemical parameters. Concentration of biochemical parameters was increased, indicating metabolic syndrome and damage to key organs in the transgenic group. In the transgenic group, the values of the triglyceride, total cholesterol, and low-density lipoprotein cholesterol significantly increased and were $2.13,1.68$, and 2.08-fold higher compared with the control group (Table I). These values were indicative of metabolic syndrome in the transgenic group. The values of ALT and AST, which reflect liver function, significantly increased and were 2.94 and 1.43-fold higher compared with the control group, respectively (Table I). The levels of uric acid and creatinine, which reflect kidney function, significantly increased and were 1.48 and 2.25-fold higher compared with the control group values, respectively (Table I). These findings revealed that transgenic mice exhibited characteristics of metabolic syndrome and that important organs involved in metabolic syndrome were damaged.

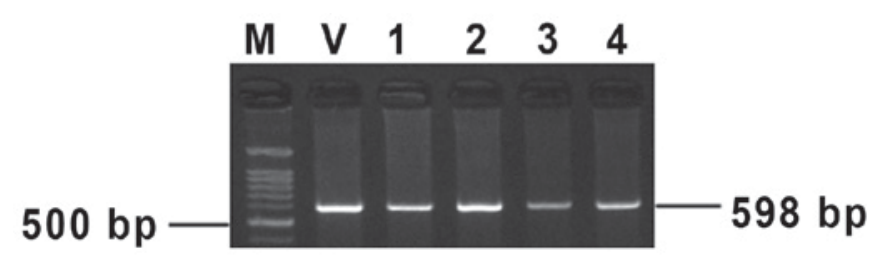

Figure 1. Identification of transgenic mice. M, DNA Ladder 100 marker; V, plasmid; lanes 1-4, transgenic mice samples.

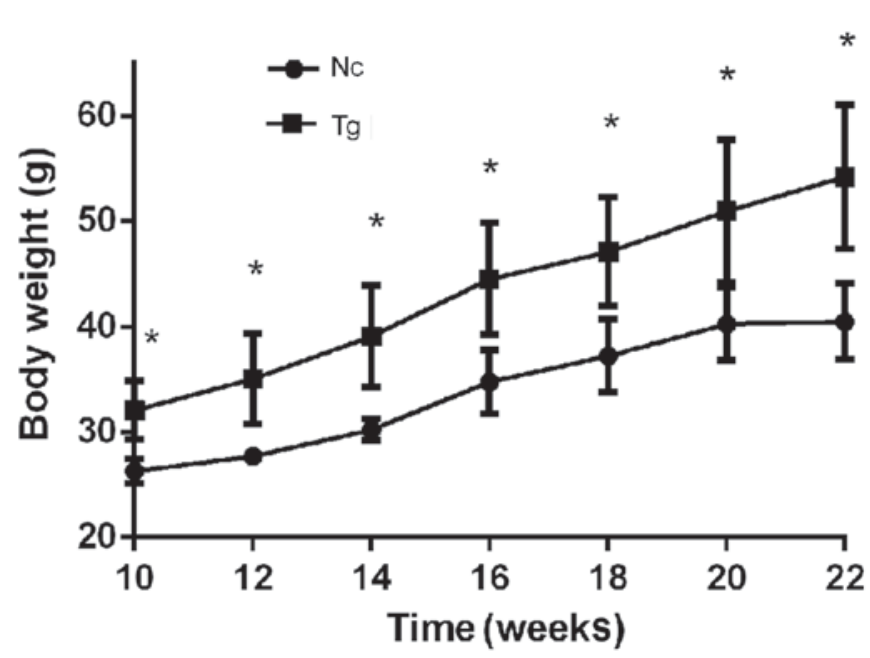

Figure 2. Average body weight of the $11 \beta-\mathrm{HSD} 1 \mathrm{Tg}$ and Nc groups with high-fat diet during 12 -weeks. ${ }^{*} \mathrm{P}<0.05$. 11 $\beta$-HSD1, $11 \beta$-hydroxysteroid dehydrogenase type $1 ; \mathrm{Tg}$, transgenic mice; $\mathrm{Nc}$, negative control.

Severely impaired glucose tolerance in transgenic mice. Fasting glucose tolerance is an important indicator that comprehensively reflects insulin release and insulin resistance in an organism. Following high-fat diet induction, the intraperitoneal glucose tolerance was quantified in the transgenic and the control groups (Fig. 3). The peak glucose of the control group appeared at 15 min after injection, whereas the peak value of the transgenic group appeared at $30 \mathrm{~min}$. The peak glucose of the control group and the transgenic group were $20.35 \pm 1.65 \mathrm{mmol} / \mathrm{l}$ and $27.03 \pm 1.49 \mathrm{mmol} / 1$, respectively $(\mathrm{P}<0.01)$. Following the peak, the blood glucose levels in these two groups began to decrease. At 4 detection points between 30 and $120 \mathrm{~min}$, the blood glucose levels in the transgenic group were all significantly higher compared with the in the control group. After $2 \mathrm{~h}$, the blood glucose level in the transgenic group remained at $14.9 \mathrm{mmol} / \mathrm{l}$, whereas the blood glucose level in the control group decreased to $8.58 \mathrm{mmol} / 1$. Therefore, after 12 weeks of high-fat diet feeding, the transgenic group exhibited severely impaired glucose tolerance. This finding is supported by the area under the glucose tolerance curve (Fig. 4), which was 1.58-fold higher for the transgenic group compared with the control group $(\mathrm{P}<0.01)$.

Significant insulin resistance in the transgenic group. Prior to feeding with a high-fat diet, the $6 \mathrm{~h}$ fasting glucose value was $10.7 \mathrm{mmol} / \mathrm{l}$ in the transgenic group and $8.28 \mathrm{mmol} / \mathrm{l}$ in the control group $(\mathrm{P}<0.01)$. Following an insulin injection, the glucose of these two groups rapidly reduced and reached a minimum after $30 \mathrm{~min}$ at $6.25 \mathrm{mmol} / \mathrm{l}$ in the transgenic group 
Table I. Biochemical parameters of the Tg and Nc groups.

\begin{tabular}{lcrr}
\hline Biochemical parameters & Tg & Nc & P-value \\
\hline ALT, IU/l & $211.33 \pm 13.47$ & $70.4 \pm 30.71$ & $<0.01$ \\
AST, IU/l & $254.67 \pm 23.80$ & $178 \pm 8.88$ & 0.04 \\
Triglyceride, $\mu \mathrm{mol} / 1$ & $0.81 \pm 0.06$ & $0.38 \pm 0.17$ & $<0.01$ \\
Total cholesterol, $\mu \mathrm{mol} / 1$ & $5.57 \pm 0.64$ & $3.31 \pm 0.85$ & 0.02 \\
HDL-C, $\mu \mathrm{mol} / 1$ & $2.23 \pm 0.13$ & $1.72 \pm 0.40$ & 0.04 \\
LDL-C, $\mu \mathrm{mol} / 1$ & $0.59 \pm 0.09$ & $0.28 \pm 0.05$ & 0.03 \\
Uric acid, $\mu \mathrm{mol} / 1$ & $269.7 \pm 10.50$ & $182.72 \pm 18.27$ & $<0.01$ \\
Creatinine, $\mu \mathrm{mol} / 1$ & $10.33 \pm 1.25$ & $4.6 \pm 1.85$ & $<0.01$
\end{tabular}

Tg, transgenic mice; Nc, negative control; ALT, alanine aminotransferase; AST, aspartate aminotransferase; HDL-C, high-density lipoprotein-cholesterol; LDL-C, low-density lipoprotein-cholesterol.

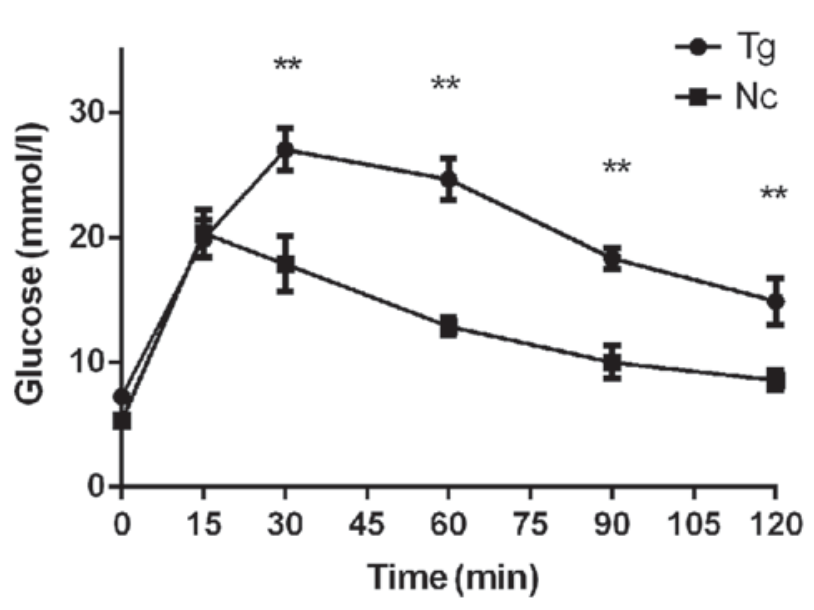

Figure 3. Intraperitoneal glucose tolerance test for the 11ß-HSD1 Tg and Nc groups; ** $\mathrm{P}<0.01$. Tg, transgenic mice; $\mathrm{Nc}$, negative control.

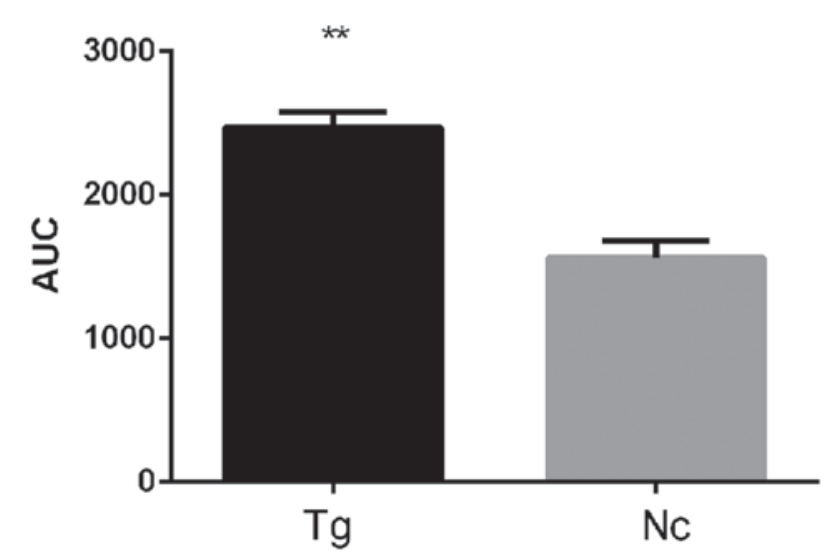

Figure 4. Glucose tolerance AUC for the 11 $\beta-\mathrm{HSD} 1 \mathrm{Tg}$ and Nc groups. ${ }^{* *} \mathrm{P}<0.01$. AUC, area under the curve; $11 \beta$-HSD1, 11 $\beta$-hydroxysteroid dehydrogenase type $1 ; \mathrm{Tg}$, transgenic mice; Nc, negative control.

and $3.73 \mathrm{mmol} / \mathrm{l}$ in the control group a significant difference was identified between these values. After $90 \mathrm{~min}$, the blood glucose levels began to increase again and reached 6.23 and $4.03 \mathrm{mmol} / \mathrm{l}$ in the transgenic group and the control group, respectively, the former was 1.55 times higher than the latter.

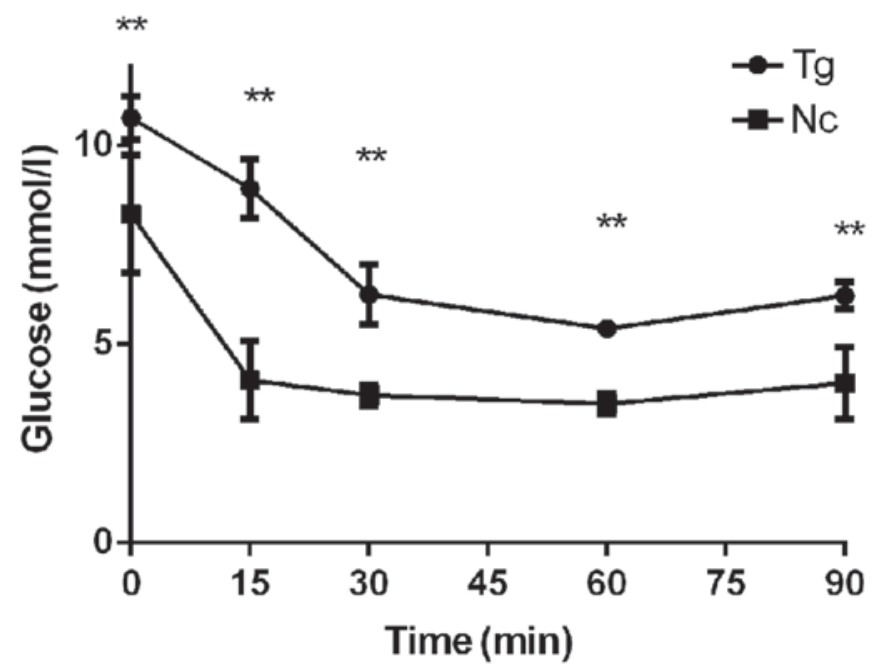

Figure 5. Insulin resistance for the $11 \beta-\mathrm{HSD} 1 \mathrm{Tg}$ and Nc groups. ${ }^{* *} \mathrm{P}<0.01$ $11 \beta$-HSD1, 11 $\beta$-hydroxysteroid dehydrogenase type 1 ; $\mathrm{Tg}$, transgenic mice; Nc, negative control.

These findings revealed that the transgenic group exhibited reduced insulin sensitivity and severe insulin resistance (Fig. 5).

Tissue expression profiles of $11 \beta-H S D 1$ in transgenic mice. The expression of $11 \beta-H S D 1$ was the highest in the liver, followed by the kidney and muscle. The pancreases did not express evident quantity of $11 \beta-\mathrm{HSD} 1$ protein. The sizes of $11 \beta$-HSD1 protein differed by tissue, and expression levels in the positive individuals were higher compared with the negative individuals (Fig. 6). The percentage of 11ß-HSD1 protein levels increased in transgenic mouse compared with the control group is presented in Fig. 7.

Severe endoplasmic reticulum stress associated with the expression of $11 \beta-H S D 1$ in the transgenic group. Endoplasmic reticulum stress is central to the development of metabolic syndrome induced by insulin resistance (15). Therefore, the present study assessed endoplasmic reticulum stress in the mice. The muscle and liver in the transgenic group exhibited 


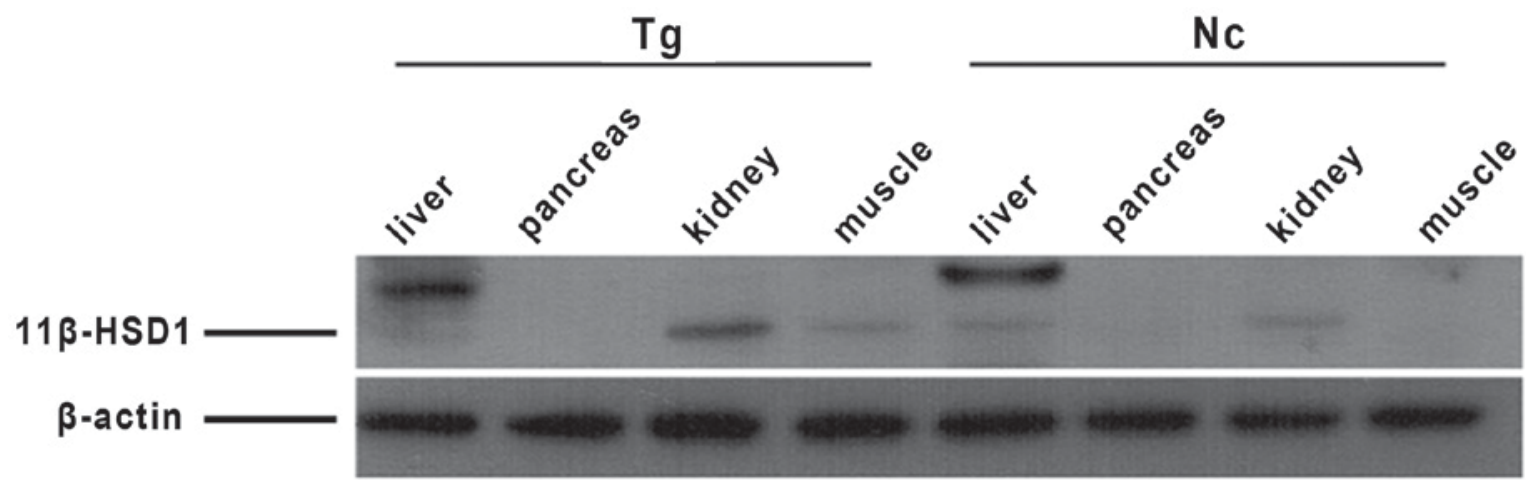

Figure 6. Tissue expression profiles of 11 $\beta$-HSD1 protein in the Tg and Nc groups. The four tissue types examined include liver, pancreas, kidney and muscle. $11 \beta$-HSD1, 11ß-hydroxysteroid dehydrogenase type $1 ; \mathrm{Tg}$, transgenic mice; $\mathrm{Nc}$, negative control.

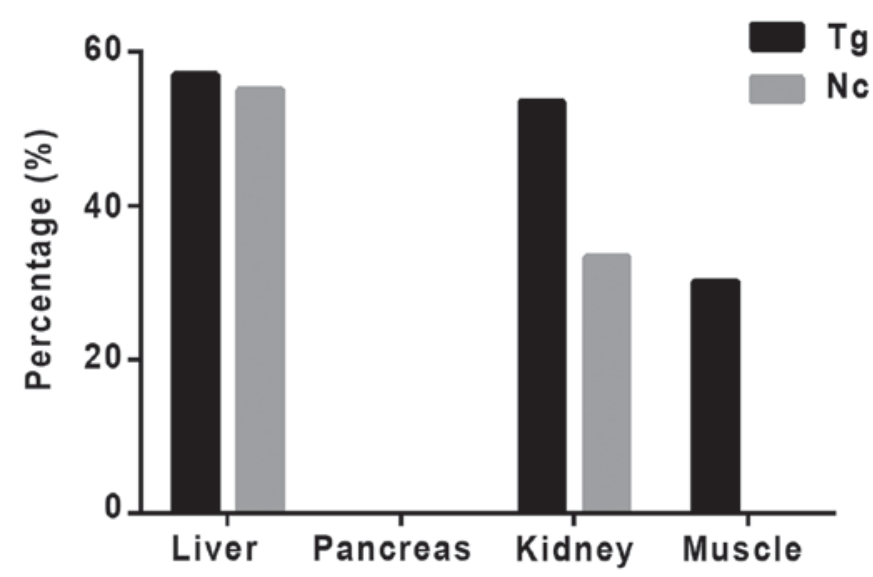

Figure 7. 11 $\beta$-HSD1 protein levels were higher in the Tg group compared

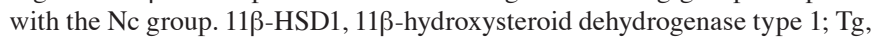
transgenic mice; Nc, negative control.

severe endoplasmic reticulum stress due to high DDIT3 expression levels, and the kidney also revealed some endoplasmic reticulum stress (Fig. 8).

Hepatic lipidosis and hepatocyte injury in transgenic mice. The liver is the largest detoxification organ in the human body and impaired liver detoxification due to hepatic lipidosis and impaired liver function are important elements in the development of metabolic syndrome, with almost two thirds of patients with type 2 diabetic having hepatic steatosis (16). Therefore, the mice liver tissues in these two groups were stained with . The it was determined that transgenic mice suffered from severe hepatic lipidosis, with lipid droplets being diffused throughout the liver and liver cells exhibiting necrosis and lysis. The sections exhibited inflammatory foci and infiltration of inflammatory cells. Conversely, the control group exhibited only mild hepatic lipidosis, hepatocytes were intact and inflammatory cell infiltration was absent (Fig. 9).

\section{Discussion}

Glucocorticoids are closely associated with metabolic syndrome, primarily by promoting gluconeogenesis, reducing insulin sensitivity in peripheral tissues, inhibiting insulin secretion and promoting islet $\beta$ cell apoptosis (6). 11 $\beta$-HSD1 is a metabolic enzyme of glucocorticoids it may activate glucocorticoids and amplify their function locally (9). The current study established transgenic mice that systemically overexpress the $11 \beta$-HSD1 gene in order to simulate the development of metabolic syndrome. After 12 weeks of high-fat diet, mice that overexpressed the $11 \beta-H S D 1$ gene exhibited obesity and significantly impaired glucose tolerance and insulin resistance. Additionally, the levels of triglycerides, total cholesterol, and high- and low-density lipoprotein cholesterol significantly increased in the transgenic mice, indicating that mice overexpressing $11 \beta-H S D 1$ exhibited characteristics of metabolic syndrome. In addition, serology measurements indicated that liver and kidney function in transgenic mice was impaired. Masuzaki et al (11) determined that the specific overexpression of $11 \beta$-HSD1 in adipose tissues may lead to increased corticosteroid levels in adipose tissues. Paterson et al (12) established transgenic mice that specifically overexpressed $11 \beta$-HSD1 in the liver. These mice exhibited mild insulin resistance, hepatic lipidosis and dyslipidemia, which was accompanied by increased hepatic lipid synthesis/efflux, elevated liver X receptor and peroxisome proliferator-activated receptor mRNA expression levels, impaired hepatic lipid clearance and significant transgenic dose-dependent hypertension combined with increased expression levels of hepatic vascular angiotensinogen (12). The findings of the present study were consistent with these previous findings.

The current study revealed that the endoplasmic reticulum stress was associated with the expression of 11 $\beta-H S D 1$ in the tissues of transgenic mice. Additionally, Kimetal(17) quantified the expression of the endoplasmic reticulum stress-associated marker genes X-box binding protein 1, activating transcription factor 4 (ATF4), ATF6 and DDIT3 (also termed CHOP) to confirm that the $11 \beta$-HSD1 inhibitor carbenoxolone attenuated tunicamycin-induced endoplasmic reticulum stress and neuronal apoptosis in the hypothalamus (17). These findings indicated that $11 \beta$-HSD1 directly induced endoplasmic reticulum stress in hypothalamic neurons and the subsequent apoptosis, which also indirectly confirmed the association between $11 \beta$-HSD1 and endoplasmic reticulum stress (17). Endoplasmic reticulum stress induced by overexpression of $11 \beta$-HSD1 activates unfolded protein response, which activates c-Jun $\mathrm{N}$-terminal kinase (JNK) via inositol-requiring enzyme-1, activated JNK may induce the serine phosphorylation of insulin receptor substrates and inhibits the normal tyrosine phosphorylation of insulin receptor substrate, which 

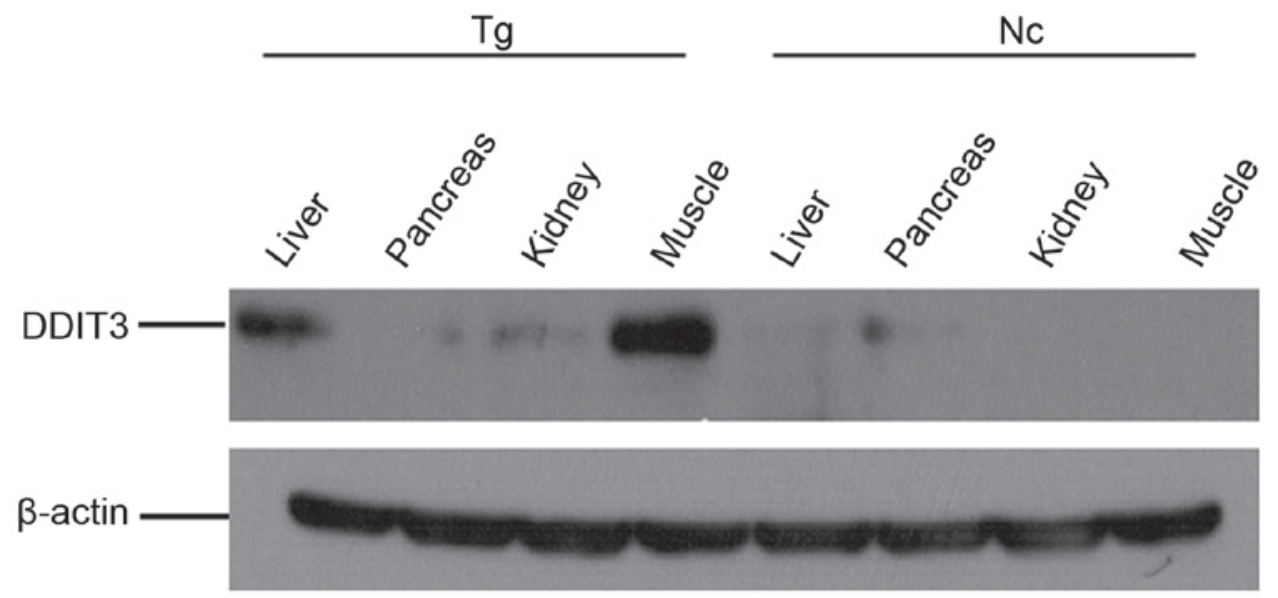

Figure 8. Tissue expression profiles of DDIT3 protein in the Tg and Nc groups. The four tissue types examined include liver, pancreas, kidney and muscle. DDIT3, DNA damage inducible transcript 3; Tg, transgenic mice; Nc, negative control.

$\mathrm{Tg}$

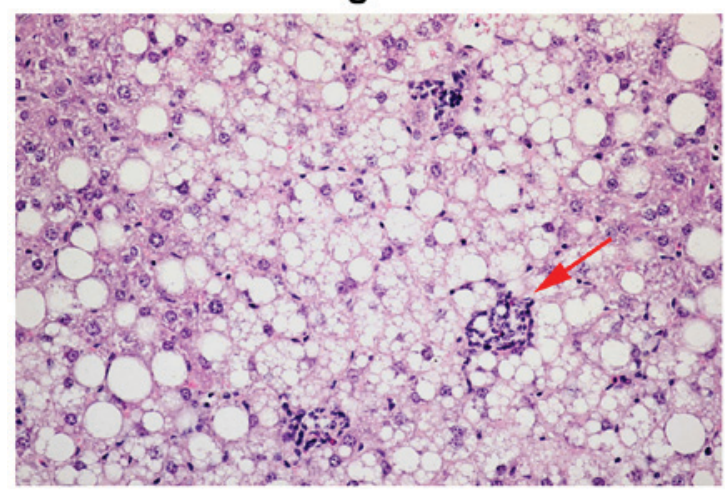

Nc

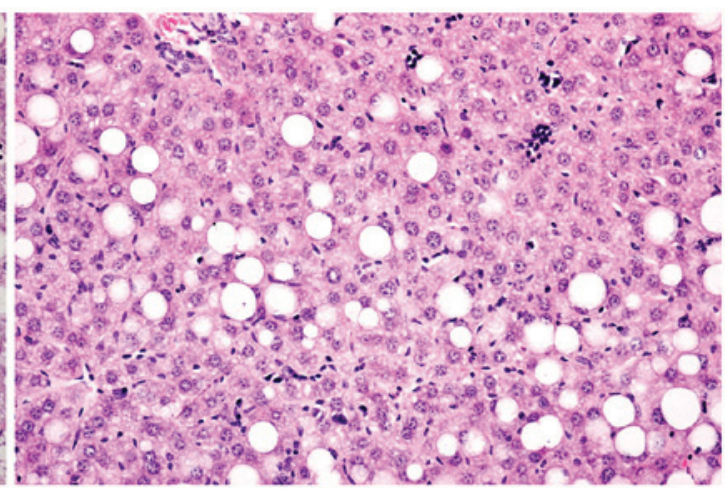

Figure 9. Histopathological changes in the mice liver of the 11 $\beta$-HSD1 Tg and Nc groups; magnification, $\mathrm{x} 40$. Red arrow indicates inflammatory foci. 11 $\beta$-HSD1, $11 \beta$-hydroxysteroid dehydrogenase type $1 ; \mathrm{Tg}$, transgenic mice; Nc, negative control.

in turn inhibit the transduction of insulin, ultimately leading to insulin resistance (15).

The present study assessed endoplasmic reticulum stress in important metabolic syndrome-associated organs. The current findings revealed that the liver and muscle tissues of mice overexpressing 11 $\beta$-HSD1 has severe endoplasmic reticulum stress and that the kidney also exhibited some endoplasmic reticulum stress. The liver is the largest detoxification organ in the human body. Impaired liver detoxification due to hepatic lipidosis and impaired liver function is an important element in the development of metabolic syndrome (18). It is possible that that the transgenic mice in the present study overexpressing $11 \beta$-HSD1 suffered from liver lesions due to their metabolic syndrome phenotype.

The quantification of liver function-associated indicators in mice revealed that the level of ALT, the most sensitive indicator of liver function, significantly increased. Additionally, the level of AST also significantly increased and was higher than the ALT level, indicating that the mouse liver parenchyma in the transgenic group was severely injured and that liver function was severely impaired. These findings were consistent with the significant endoplasmic reticulum stress observed in the liver. Histological evaluation identified severe fat deposition, lipid droplets that had diffused throughout the liver, necrotic and lysed hepatocytes, many inflammatory foci and significant inflammatory cell infiltration in the livers of transgenic mice. However, endoplasmic reticulum stress-induced hepatocyte apoptosis may be directly caused by high $11 \beta$-HSD1 expression in the liver or indirectly caused by $11 \beta$-HSD 1 -induced lipid deposition in the liver and this mechanism requires further investigation.

In conclusion, the present study successfully established transgenic mice that systemically overexpressed $11 \beta-H S D 1$. These mice exhibited obvious characteristics of metabolic syndrome, such as severe hepatic lipidosis, hepatocyte necrosis, impaired liver function, and endoplasmic reticulum stress in muscle, liver and kidney. These findings indicate that the systemic overexpression of $11 \beta$-HSD1 in mice produces pathological changes that approximate to the clinical symptoms of human metabolic syndrome. Therefore, these mice may be used as a model for future studies of metabolic syndrome.

\section{Acknowledgements}

The current study was supported by The National Natural Science Foundation of China (grant no. 31372276), The National Basic Research Program of China (grant no. 2015CB943100), National Science and Technology Major Project (grant 
no. 2016ZX08006-001), The State Key Laboratory of Animal Nutrition (grant no. 2004DA125184G1602) and The Agricultural Science and Technology Innovation Program (grant nos. ASTIP-IAS05 and ASTIP-IAS-TS-4).

\section{References}

1. Alberti KG, Eckel RH, Grundy SM, Zimmet PZ, Cleeman JI, Donato KA, Fruchart JC, James WP, Loria CM, Smith SC Jr, et al: Harmonizing the metabolic syndrome: A joint interim statement of the International Diabetes Federation Task Force on Epidemiology and Prevention; National Heart, Lung and Blood Institute; American Heart Association; World Heart Federation; International Atherosclerosis Society; and International Association for the Study of Obesity. Circulation 120: 1640-1645, 2009.

2. Thomas GN, Ho SY, Janus ED, Lam KS, Hedley AJ and Lam TH; Hong Kong Cardiovascular Risk Factor Prevalence Study Steering Committee: The US National Cholesterol Education Programme Adult Treatment Panel III (NCEP ATP III) prevalence of the metabolic syndrome in a Chinese population. Diabetes Res Clin Pract 67: 251-257, 2005.

3. Aguilar M, Bhuket T, Torres S, Liu B and Wong RJ: Prevalence of the metabolic syndrome in the United States, 2003-2012. Jama 313: 1973-1974, 2015.

4. Sookoian S and Pirola CJ: Metabolic syndrome: From the genetics to the pathophysiology. Curr Hypertens Rep 13: 149-157, 2011.

5. Eckle RH, Grundy SM and Zimmet PZ: The metabolism syndrome. Lancet 365: 1415-1428, 2005.

6. Lee MJ, Pramyothin P, Karastergiou K and Fried SK: Deconstructing the roles of glucocorticoids in adipose tissue biology and the development of central obesity. Biochim Biophys Acta 1842: 473-481, 2014.

7. Seckl JR and Walker BR: Minireview: 11beta-hydroxysteroid dehydrogenase type 1 a tissue-specific amplifier of glucocorticoid action. Endocrinology 142: 1371-1376, 2001.

8. Alberts P, Nilsson C, Selen G, Engblom LO, Edling NH, Norling S, Klingström G, Larsson C, Forsgren M, Ashkzari M, et al: Selective inhibition of 11 beta-hydroxysteroid dehydrogenase type 1 improves hepatic insulin sensitivity in hyperglycemic mice strains. Endocrinology 144: 4755-4762, 2003.
9. Andrew R, Westerbacka J, Wahren J, Yki-Järvinen $\mathrm{H}$ and Walker BR: The contribution of visceral adipose tissue to splanchnic cortisol production in healthy humans. Diabetes 54: 1364-1370, 2005.

10. Wang L, Liu J, Zhang A, Cheng P, Zhang X, Lv S, Wu L, Yu J, Di W, Zha J, et al: BVT.2733, a selective 11beta-hydroxysteroid dehydrogenase type 1 inhibitor, attenuates obesity and inflammation in diet-induced obese mice. PLoS One 7: e40056, 2012.

11. Masuzaki H, Paterson J, Shinyama H, Morton NM, Mullins JJ, Seckl JR and Flier JS: A transgenic model of visceral obesity and the metabolic syndrome. Science 294: 2166-2170, 2001.

12. Paterson JM, Morton NM, Fievet C, Kenyon CJ, Holmes MC, Staels B, Seckl JR and Mullins JJ: Metabolic syndrome without obesity: Hepatic overexpression of 11beta-hydroxysteroid dehydrogenase type 1 in transgenic mice. Proc Natl Acad Sci USA 101: 7088-7093, 2004.

13. Tebbe CC and Vahjen W: Interference of humic acids and DNA extracted directly from soil in detection and transformation of recombinant DNA from bacteria and a yeast. Appl Environ Microbiol 59: 2657-2665, 1993.

14. Li L, Zhao Z, Xia J, Xin L, Chen Y, Yang S and Li K: A Long-Term High-Fat/High-Sucrose Diet Promotes Kidney Lipid Deposition and Causes Apoptosis and Glomerular Hypertrophy in Bama Minipigs. PLoS One 10: e0142884, 2015.

15. Ozcan U, Cao Q, Yilmaz E, Lee AH, Iwakoshi NN, Ozdelen E, Tuncman G, Görgün C, Glimcher LH and Hotamisligil GS: Endoplasmic reticulum stress links obesity, insulin action, and type 2 diabetes. Science 306: 457-461, 2004.

16. Targher G, Bertolini L, Padovani R, Rodella S, Tessari R, Zenari L, Day C and Arcaro G: Prevalence of nonalcoholic fatty liver disease and its association with cardiovascular disease among type 2 diabetic patients. Diabetes Care 30: 1212-1218, 2007.

17. Kim J, Jung EJ, Moon SS and Seo M: Protective effect of carbenoxolone on ER stress-induced cell death in hypothalamic neurons. Biochem Biophys Res Commun 468: 793-799, 2015.

18. Nannipieri M, Gonzales C, Baldi S, Posadas R, Williams K, Haffner SM, Stern MP and Ferrannini E; Mexico City diabetes study: Liver enzymes, the metabolic syndrome, and incident diabetes: The Mexico City diabetes study. Diabetes Care 28: $1757-1762,2005$ 